\title{
Abattoir-based prevalence of avian tuberculosis in chicken slaughtered at Poultry abattoir in Bishoftu, Central Ethiopia
}

\author{
Gezahegne Mamo
}

Department of Veterinary Microbiology, Immunology, Public Health, College of Veterinary Medicine and Agriculture, Addis Ababa University, P.O.Box 34, Bishoftu, Ethiopia gezahegne.mamo@ aau.edu.et; Tel. +251-911-678195

\begin{abstract}
A cross-sectional abattoir-based study was conducted in apparently healthy chicken in Bishoftu town, Ethiopia to estimate the prevalence of avian tuberculosis and isolate its causative agent. The occurrence of avian tuberculosis was investigated using postmortem examination, bacteriological culture and acid-fast staining methods. Of the total 648 chicken examined to detect avian tuberculosis, 42 tissue samples showing gross pathological tuberculous-like lesions were collected from liver, spleen and intestine. The overall prevalence of avian tuberculosis in poultry was $6.48 \%(42 / 648)(95 \% \mathrm{CI}: 4.53-8.38)$ on the basis of detailed postmortem examination. Out of 42 tissue samples cultured on Lowenstein-Jensen medium, 14 (33.3\%) were bacteriologically culture positive and showed growth of dough-shaped smooth colony characteristic and out of these 14 culture positive samples, 5 (35.7\%) were acid-fast positive mycobacteria. Statistical significant difference was observed in the prevalence of avian TB among chicken with different body condition scoring $\left(\mathrm{x}^{2}=23.593, \mathrm{p}\right.$ $=0.001$ ). Multivariable logistic regression analysis for risk factors showed that body condition scoring has a high statistical significant association with the prevalence of avian tuberculosis in the study area $(\mathrm{p}<0.05)$. Poor body conditioned chicken were more likely to show TB lesions (OR=4.45, 95\% CI, 2.338.52) than good body conditioned chicken. The present preliminary study on avian TB using postmortem lesion examination and microbiological methods revealed the occurrence of avian TB in low prevalence in apparently healthy chicken originated from intensive poultry farms in Bishoftu area; hence detail poultry meat inspection should be practiced at poultry abattoirs in order to reduce the public health risk.
\end{abstract}

Keywords: Abattoir, Avian tuberculosis, Postmortem examination, Poultry, Ethiopia 


\section{Introduction}

Ethiopia has large population of chicken, estimated to be 56.53 million, of which local breeds representing $94.31 \%$, hybrid chicken $3.21 \%$ and exotic breeds of chicken 2.49\% (CSA, 2017). As in most developing countries, in Ethiopia village chickens raised under backyard production system make up the largest proportion, more than $95 \%$, while chicken in commercial production system constitute the remaining small proportion of the national population (Dessie et al., 2003; Habte et al., 2017).

Poultry sector has an important contribution in the provision of high quality protein food in the form of meat and egg to rural smallholder farming families in Africa (Sonaiya et al., 1999) and Ethiopia (Dessie and Ogle 2001). Even though the backyard poultry production system constitutes the majority of poultry production in Ethiopia, currently large and small-scale commercial poultry farms are expanding in urban and periurban areas of Ethiopia to meet the poultry meat and egg demand of the fast growing urban population of the country. However, this growing poultry sector is constrained by various factors including infectious diseases which directly or indirectly influence the productivity of the sector (Habte et al., 2017).

Avian tuberculosis (Avian TB) is one of the important infectious diseases of various species of birds including domestic chicken, pet birds, free-living and captive wild birds, and it has also public health significance (Dhama et al., 2011). Avian tuberculosis in poultry is most often caused by Mycobacterium avium subsp avium. However, more than ten mycobacterial species including Mycobacterium genavense, $M$. avium subsp. hominissuis, $M$. intracellulare, $M$. scrofulaceum, $M$. fortuitum and other potential pathogenic mycobacterial species have been reported to infect birds (Tell et al., 2003; Shivaprasad, and Palmieri, 2012; OIE, 2018).

The oral route of infection appears to be the primary mode of transmission of avian TB in birds and majority of the lesions were detected in the intestine and liver (OIE, 2018). In most cases, infected birds show no clinical signs, but they may eventually become lethargic and emaciated. Many affected birds show diarrhea along with marked atrophy of breast muscle, and comb and wattles may regress and become pale. Under intensive husbandry conditions, sudden death may occur, often associated with severe lesions in the liver; such lesions are easily observed at post-mortem examination (Tell et al., 2001). Unlike TB 
in animals and man, lesions in lungs of birds are rare. Tubercular nodules can be seen in liver, spleen, intestine and bone marrow. Granulomatous lesion without calcification is a prominent feature. If typical lesions of tuberculous are present at necropsy, demonstration of acid-fast bacilli in smears or histopathologic sections made from affected organs is regarded as sufficient for positive diagnosis (OIE, 2018).

In Ethiopia, in spite of the existence of large population of chicken and potential future expansion of the poultry industry, infectious diseases such as avian TB has not been well studied. Few avian TB studies carried out so far on traditionally managed local chicken reported a prevalence of $6.3 \%$ in central Ethiopia (Tadesse et al., 2004) and 4.23\% in Shashemene district of west Aris zone of Oromia Region (Abda et al., 2015). However, there is no published abattoirbased avian TB study on slaughtered exotic chicken originated from intensive commercial production system in Ethiopia. Therefore, the present study was designed to investigate the occurrence of avian tuberculosis based on pathological lesions and isolation of its causative agents in apparently healthy chicken slaughtered at a poultry abattoir in Bishoftu, central Ethiopia.

\section{Materials and methods}

\section{Study Area}

The study was conducted at Alema poultry abattoir located in Bishoftu town in East Shoa Zone of Oromia Regional State from October 2015 to June 2016. Bishoftu town is located at 47 kilometers south east of Addis Ababa. The area is located at $9^{\circ} \mathrm{N}$ latitude and $40^{\circ} \mathrm{E}$ longitude at an altitude of 1850 meters above sea level in central high lands of Ethiopia. In Bishoftu, there are few private large scale commercial poultry farms and a number of small-scale poultry farm. Alema intensive poultry farms is the second largest commercial poultry farms in the country next to ELFORA poultry farm delivering nearly half a million broilers to Addis Ababa market each year (Abera, 2018). This farm supplies broiler chicken for slaughter to Alema poultry abattoir, which is one of the modern poultry abattoirs in Ethiopia and is producing chicken meat for local market mainly to supermarkets and hotels in Addis Ababa and its surrounding. 


\section{Study animals}

The study was carried out on exotic chickens which were brought for slaughter to Alema poultry abattoir. The chickens were apparently healthy raised under intensive commercial farming system supplied with formulated feed ration and the chickens obtained vaccination for major poultry diseases such as Newcastle disease and fowl cholera. The study animals were male and female of different body condition and the same age group.

\section{Study design}

A cross-sectional study design was carried out primarily to estimate the apparent prevalence of avian TB based on the presence of suspected TB lesions during postmortem examination of the slaughtered chicken in the poultry abattoir. The organs with suspected gross pathological lesions particularly liver, spleen and intestine were examined using detail postmortem examination method.

\section{Sample size determination}

The determination of sample size for this study was calculated by considering $6.3 \%$ expected prevalence as reported in previous avian tuberculosis study in backyard chicken of central Ethiopia (Tadesse et al., 2004), 95\% confidence interval and 5\% required precision, and using the formula for estimation of sample size as given below according to Thrusfield (2007).

$$
n=\frac{1.96^{2} \mathrm{XP}_{\exp } \mathrm{X}\left(1-\mathrm{P}_{\mathrm{exp}}\right)}{\mathrm{d}^{2}}
$$

Where $n=$ required sample size, $d=$ desired absolute precision, $\mathrm{P}_{\exp }=$ expected prevalence

Thus, the calculated sample size was 91 . However, as the expected prevalence considered in the sample size calculation was from the previous study done on local chicken in backyard scavenging management system the author believed the existence of major differences from the present abattoir-based avian TB study which targeted on apparently healthy exotic breeds managed under intensive production system. Therefore, $50 \%$ expected prevalence was considered and the final calculated samples size was 384 and in order to increase the precision of the study a total of 648 animals were examined. 


\section{Sampling methods}

A systematic random sampling procedure was used to pick every sixth chicken slaughtered during the visit. The abattoir was visited for four days per week depending on the regular schedule of the abattoir to collect the samples until the sample size was fulfilled. During the study on average 200-300 chickens were slaughtered per day based on local market needs. Information about the flock of chickens slaughtered in each visit days with regarding age, breed, flock health status including vaccination history; farm of origin, management system were recorded.

\section{Study Methodology}

Ante mortem examination

Ante-mortem examination was done after the chickens were hung in shackles and before bleeding occurred and individual chicken was examined visually for general condition including the presence of visible abnormalities and superficial lesions. Body condition scoring for each chicken was done as poor body condition (Score 0) and good body condition (Score 1) according to Gregory and Robins (1998).

\section{Gross postmortem examination and tissue sampling}

A total of 648 chickens were examined for suspected gross pathological lesions and tissue samples were taken from chickens with suspected pathological TB lesions. Postmortem examinations were done on each chicken carcass to detect the presence of tuberculous lesion in different visceral organs with particular emphasis on liver, intestine and spleen. During post-mortem examination, gross lesions were sliced using separate sterile surgical blades and the lesion were described grossly on characteristic and distribution of the lesion. The suspected lesions were sampled and kept in labeled universal bottle containing phosphate buffered saline solution and transported in ice box under cold chain condition to Aklilu Lemma Institute of Pathobiology (ALIPB) laboratory for mycobacterial culture isolation. 
Tissue processing for Mycobacterium culture

Isolation of mycobacteria from tissues was done in accordance with OIE (2018) protocol. Approximately 3-5 grams of tissue samples from suspected TB lesions were sectioned into pieces using sterile blades, and then homogenized by pestle and mortar. The homogenate were decontaminated by adding an equal volume of $4 \% \mathrm{NaOH}$ followed by centrifugation at $3000 \mathrm{rpm}$ for 15 minutes. The supernatant were discarded while the sediment was neutralized by $1 \%(0.1 \mathrm{~N}) \mathrm{HCl}$ using phenol red as an indicator. Neutralization was achieved when the color of the solution changed from purple to yellow. Thereafter, $0.1 \mathrm{ml}$ of suspension from each sample was spread onto a slope of Lowenstein-Jensen (L-J) medium. Duplicates of L-J were used; one enriched with 1\% sodium pyruvate while the other was enriched with glycerol. Culture tubes were kept inclined with loosened screw to facilitate the evaporation of excess moisture and inoculum fluid for one week. After one week the tubes were placed vertical with tightened screw and incubated aerobically at $37^{\circ} \mathrm{C}$ for 16 weeks or until macroscopic growth was observed while they were examined on a regular basis for colony growth. Cultures were first examined after $8^{\text {th }}$ week of inoculation and subsequently every week up to 16 weeks for the presence of any growth. No evidence of bacterial growth after this incubation period was considered as a negative result. A culture was considered positive when white spot colonies were seen. Growths of Mycobacterium avium colonies were confirmed by Ziehl-Neelsen (Acid-fast) staining and its long incubation period. Whenever, colonies were seen, sub-culturing and acid-fast staining were performed to confirm the presence of acid fast bacilli.

\section{Acid-Fast Staining Technique}

The colonies which grow on the L-J media were stained with Ziehl-Neelsen method for detection of acid-fast bacilli according to Quinn et al. (1994). Stained slides were observed using oil immersion of $100 \mathrm{x}$ lens objective of a microscope. Each slide was examined for 30 minutes to detect acid-fast bacilli in the examined microscopic fields. The findings were recorded according to the bacteria appearance in which, observation of a bacilli with pink rod-shaped appearance taken as positive while if no acid-fast bacilli were observed, the sample was regarded as negative for acid-fast bacilli. 


\section{Data management and analysis}

The data collected from abattoir and laboratory examination were entered and coded into Microsoft excel spread sheets and analyzed by STATA statistical software version 11 (STATA Corp. College station, TX). The association between different risk factors with the occurrence of avian tuberculosis suspected postmortem lesions were analyzed using chi-square $\left(x^{2}\right)$ and multivariable logistic regression. A p-value $<0.05$ was considered statistically significant and in estimating the effect of different risk factors in terms of odds ratio (OR) with corresponding $95 \%$ confidence interval, statistical significance was assumed if the confidence interval did not include one among its values.

\section{Results}

\section{Prevalence of avian tuberculosis and associated risk factors}

The prevalence of avian TB at Alema poultry abattoir was $6.48 \%$ (95\% CI: 4.58 to $8.38 \%$ ) based on postmortem examination for the presence of suspected gross pathological TB-like lesions. The prevalence of the disease varies with different risk factors. From the total chicken examined in the abattoir, 56.2\% were female and $43.8 \%$ were male. The multivariable logistic regression analysis for the association between prevalence and various risk factors is presented in Table 1 . The analysis showed that chicken with poor body condition were more likely to be positive for avian TB suspected lesions as compared to chicken with good body condition and the difference was statistically significant (adjusted $\mathrm{OR}=4.46 ; 95 \% \mathrm{CI}: 2.33-8.53$ ). The association of other risk factor (sex of the chicken) with the prevalence of avian TB was not statistically significant $(p>0.05)$.

Table 1. Multivariable logistic regression analysis of the effect of different risk factors on the prevalence of TB in poultry.

\begin{tabular}{llccccc}
\hline Variables & categories & $\begin{array}{l}\text { Total No. } \\
\text { of chicken } \\
\text { examined }\end{array}$ & $\begin{array}{l}\text { Positive } \\
\text { for TB } \\
\mathbf{( \% )}\end{array}$ & $\begin{array}{l}\text { Crude OR } \\
\mathbf{( 9 5 \% ~ C I )}\end{array}$ & $\begin{array}{l}\text { Adjusted } \\
\text { OR } \\
\mathbf{( 9 5 \%} \text { CI) }\end{array}$ & p-Value \\
\hline $\begin{array}{l}\text { Chicken } \\
\text { sex }\end{array}$ & Male & 287 & $20(6.97 \%)$ & 1 & 1 & 0.641 \\
& Female & 361 & $22(6.1 \%)$ & $\begin{array}{c}0.88(0.43- \\
1.62)\end{array}$ & $\begin{array}{c}0.86(0.45- \\
1.63)\end{array}$ & \\
$\begin{array}{l}\text { Body } \\
\text { condition }\end{array}$ & Good & 188 & $16(3.5 \%)$ & 1 & 1 & 0.000 \\
& Poor & 460 & $26(13.8)$ & $\begin{array}{c}4.45(2.33- \\
8.53)\end{array}$ & $\begin{array}{c}4.46(2.33- \\
8.53)\end{array}$ & \\
\hline
\end{tabular}

Ethiop. Vet. J., 2020, 24 (1), 1-14 


\section{Antemortem findings}

Antemortem examination of all the 648 chickens showed no clinical signs of any diseases and the chicken were apparently healthy. Their live body weight was estimated to range from $0.6-1 \mathrm{~kg}$ in which 460 chickens $(71 \%)$ were in poor body condition status, and 188 chickens (29\%) were in good body condition status.

\section{Gross pathological postmortem findings}

During detail examination of the organs from the 648 chickens, gross lesions were observed in liver, spleen and intestine and more typical tuberculous lesions were seen in liver. The lesions were grayish-yellow to grayish-white, pinpoint to irregularly round, and few were nodules measuring up to $2 \mathrm{~mm}$ in diameter were swollen above the surface of the affected organs. Calcification was not seen in the nodules. Spleen and liver with pathological lesion were enlarged in size. Grossly, the lesions on liver were characterized as small; multiple circular nodules with light-yellowish color with white spot appear up on dissection with no calcification seen in the nodules (Figure 1).

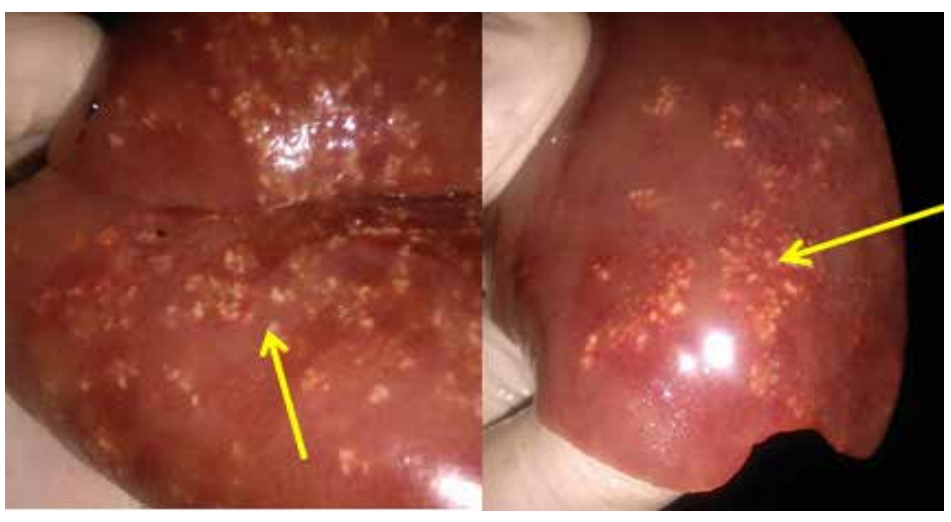

Figure 1. Small light yellowish nodular tuberculous-like lesions (yellow arrows) on the liver of chicken found during postmortem examination.

Regarding the distribution of lesions in different organs, larger proportion $(88.10 \%)$ of the lesions were observed in liver, followed by spleen $(9.52 \%)$ and intestine (2.38\%) (Table 2). In most of the chickens that have manifested gross lesions, more than one type of organs was affected. 
Table 2: Association of gross pathological lesions of suspected avian tuberculosis with distribution of the lesions in different organs of the examined chicken

\begin{tabular}{lccccc}
\hline Organs & $\begin{array}{l}\text { Sample } \\
\text { examined }\end{array}$ & $\begin{array}{l}\text { Sample } \\
\text { positive }\end{array}$ & \% & $\mathbf{X}^{\mathbf{2}}$ - value & p-value \\
\hline Liver & 41 & 37 & 88.10 & 10.43 & 0.108 \\
Spleen & 35 & 4 & 9.52 & & \\
Intestine & 28 & 1 & 2.38 & \\
Total & 104 & 42 & 100 & \\
\hline
\end{tabular}

\section{Isolation of Mycobacteria from suspected TB-like lesions}

From the 42 suspected TB-like lesions samples cultured for mycobacteria, growth of Mycobacterium was observed in 14 samples on pyruvate enriched L-J culture media after 3 weeks of incubation. The colonies were smooth, light yellowish and slightly raised colony. The origins of samples for the 14 isolates were liver, spleen and intestinal tissue. The culture isolates were further identified by conducting acid-fast staining and five isolates were confirmed to be acid-fast by Ziehl-Neelsen staining and they were stained bright red (pink) and had rod-shaped morphology (Figure 2).

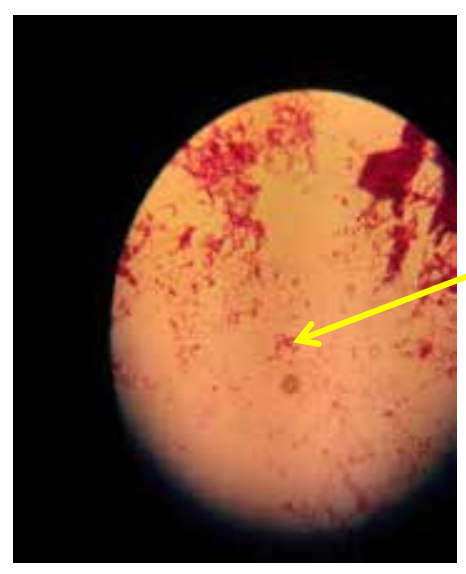

Figure.2 Detection of acid-fast bacilli of Mycobacterial culture positive samples from suspected avian TB chicken. The acid-fast bacilli were pink (indicated by yellow arrow), straight or slightly curved rods, occurring singly or in small groups in stained smears examined microscopically (oil-immersion magnification 1000X). 


\section{Discussion}

In the present study an attempt was made to estimate the prevalence of avian tuberculosis based on abattoir-based postmortem examination of suspected TB-like lesions and identify the associated risk factors in chicken slaughtered at modern poultry abattoir in Bishoftu town. The study showed that the prevalence of avian TB in slaughtered exotic breeds of chicken from intensive poultry farm was low (6.48\%) based detection of suspected TB-like lesions and the result was in agreement with the finding of previous study (Tadesse et al., 2004) with $6.3 \%$ prevalence in Adama town from apparently healthy local chickens and (Abda et al., 2015) with 4.23\% prevalence in local chickens at Shashemene district. So far, no published report of avian tuberculosis in abattoir based on postmortem examination of suspected TB-lesions in exotic chicken originated from intensive commercial poultry farms in Ethiopia.

In the present study, the majority of the chickens infected had poor body condition and there was a significant association of avian TB prevalence with body condition of chickens and a number of studies indicated the association of poor body condition with avian TB prevalence (Dhama et al., 2008, Miguel, 2012; Abda et al., 2015). Thus, chicken with poor body condition in a farm or in antemortem inspection could be suggestive of avian tuberculosis. These studies indicated that chickens are constantly exposed to overcrowding (which may lead to stress), and unhygienic external environments may serve as sources of infection. Overcrowding within a flock can result a stressful condition for the chickens, which in turn could affect the nature and number of lesions occurring (Tell et al., 2001; Fulton and Thoen, 2003; OIE, 2018). Based on postmortem examination of the TB-like lesions, majority $(88.10 \%)$ of the suspected gross TB-like lesions were localized in liver and the rest were localized in spleen and intestine which might suggest that the most probable route of infection was oral route through ingestion. The route of infection helps to explain the low incidence of pulmonary lesions in birds when compared to mammals in which the lung is infected first (Gonzalez et al., 2002).The overall characteristics of gross lesions and its distribution in different organs which were found in this study were in line with the previous findings (Tadesse et al., 2004). The variation in size and number of lesions recorded in chickens could also be caused by successive episodes of reinfection from previously established lesions (Thoen, 1997).

In mycobacteriological culture examination, fourteen isolates were identified as positive for Mycobacterium as suspected by colony morphology and growth 
characteristics; and five of the isolates were acid-fast positive as confirmed by acid-fast staining of the bacilli. Typically, species like $M$. avium produces "smooth" colonies which are virulent for chickens while variants with smooth domed or rough colonies are avirulent (Fulton and Thoen, 2003; Dhama et al., 2007). The recovery of Mycobacterium in culture growth was low in proportion, $33.3 \%(14 / 42)$ and this could be due to the non-optimal condition of the culture medium for non-tuberculous mycobacteria species including $M$. avium as this study used the available L-J media for culture of the isolates. Similar studies have also reported a low culture recovery rate for non-tuberculosis mycobacteria isolation using LJ media (Mamo et al., 2011; Abda et al., 2015). On the other hand, the low culture positivity might also be due to the absence of viable mycobacteria in necrotized TB-like lesion in which incompletely necrotic lesions, tubercle bacilli are dead and therefore no growth could be detected up on culture on LJ media (Quinn et al., 1994).

In the present study, analysis of tissues samples that resulted characteristics growth of colony of suspected Mycobacterium avium using acid-fast staining technique revealed that out of 42 samples only five (11.9\%) were acid-fast positive. Even though, acid-fast staining method can be used for diagnosis of avian tuberculosis combined with clinical sign and mycobacterial isolation it has low sensitivity (Garg et al., 2003; Dhama et al., 2007; Zhu et al., 2018). Diseases causing nodular lesions such as leucosis, pseudotuberculosis and coligranulomas can be considered as differential diagnosis in those samples with negative acid-fast staining results (Dhama et al., 2011).

\section{Limitation of the study}

This study was carried out in abattoir setting where there might be a possibility of contamination from the environment as the group in $M$. avium are ubiquitous. The other main limitation of the study was the isolates were not further analyzed by molecular method to confirm the causative agent of avian TB, $M$. avium subsp. avium using molecular techniques.

\section{Conclusion}

In general, in Ethiopia there is limited information on epidemiology of avian TB and its associated risk factors in exotic chicken reared under intensive commercial poultry production system. Hence, the present abattoir-based study revealed the occurrence of avian tuberculosis in apparently healthy exotic 
chickens which originated from intensive commercial poultry farm in Bishoftu area and chicken with poor body condition were associated with the finding of suspected TB lesions; hence, it seems appropriate to recommend during antemortem inspection chicken with poor body condition should be isolated and examined thoroughly. Moreover implementation of routine detailed poultry meat inspection procedures in poultry abattoirs is important to detect the pathological lesions and produce safe poultry meat for consumers. The finding of higher proportion of TB-like lesions which were negative in culture and acidfast staining warrants the need further investigation on the causative agents of the pathological lesions other than avian tuberculosis.

\section{Acknowledgements}

The author would like to acknowledge Prof. Gobena Ameni and his research team for providing valuable support and assistance during the research work and mycobacterial culture at Aklilu Lemma Institute of Pathobiology. The author also expresses deep gratitude to Alema Poultry abattoir and its staffs for their positive cooperation during data collection by undergraduate and graduate students involved in sub-thematic research "Paratuberculosis and avian tuberculosis in livestock of Ethiopia".

\section{Conflict of Interest}

The author has declared that no conflict of interests exists.

\section{Funding}

This study was financially supported by the Thematic research project entitled: "Mycobacterial infection in Ethiopia: sub-thematic research paratuberculosis and avian tuberculosis in livestock of Ethiopia" funded by Addis Ababa University. The funding organization had no any role in the design of the study and collection, analysis, and interpretation of data and in writing the manuscript.

\section{References}

Abda, S., Mamo, G., Worku, A., Ameni, G., 2015. Preliminary study on avian tuberculosis and associated risk factors in domestic chickens at Shashemene district, Ethiopia. J. Biol. Med. Sci., 3, 13-23. 
Abera, A., 2018. Review on Chicken Production in Ethiopia with emphasis on Meat Production, Munich, GRIN Verlag, https://www.grin.com/document/385849.

CSA, 2017. Central Statistics Authority (CSA): Agricultural sample survey 2016/17. Report on livestock and livestock characteristics. Statistical Bulletin No. 585. Addis Ababa, Ethiopia.

Dhama, K., Mahendran, M. \& Tomar, S., 2007. Avian tuberculosis: an overview. Poultry Punch, 24 (3), 38-52.

Dhama, K., Mahendran, M., \& Tomar, S., 2008. Pathogens transmitted by migratory birds: threat perceptions to poultry health and production. Int. J. Poult. Sci., 7 (6), 516-525.

Dhama, K., Mahendran, M., Tiwari R., Singh S.D., Kumar D., Singh S., Sawant P.M., 2011. Tuberculosis in birds: insights into the M. avium infections: review article, Vet. Med. Int, Vol 2011, 1-13. doi:10.4061/2011/712369

Fulton, R. and Thoen, C., 2003. Tuberculosis. Diseases of Poultry, Iowa State University Press, USA. Pp. 836-844.

Gonzalez, M., Rodriguez-Bertos, A., Gimeno, I., 2002. Outbreak of avian tuberculosis in 48-week-old commercial layer hen flock. Avi. Dis., 46, 1055-1061.

Garg, S.K, Tiwari, R.P., Tiwari, D., Singh, R., Malhotra, D., Ramnani, V.K., et al., 2003. Diagnosis of tuberculosis: available technologies, limitations, and possibilities. $J$. Clin. Lab. Ana.l, 17, 155-63.

Habte, T., Amare, A., Bettridge, J., Collins, M., Christley, R., Wigley P., 2017. Guide to chicken health and management in Ethiopia, ILRI manual 25. Nairobi, Kenya: International Livestock Research Institute (ILRI), Pp. 17-44.

Mamo, G., Bayleyegn, G., Sisay T., Legesse, M., Medhin, G., Bjune, G., Abebe, F. \& Ameni, G., 2011. Pathology of Camel Tuberculosis and Molecular characterization of Its Causative Agents in Pastoral Regions of Ethiopia. PLoS One, 6(1), e15862.

Miguel, D.S., 2012. Mycobacteriosis: An issue of veterinary clinics: Exotic Animal Practice. Vet. Clin. N. Am.,15 (1), 1-10.

OIE, 2018. Avian tuberculosis. OIE _Terrestrial Manual 2018: Office International des Epizooties (OIE) Chapter 3.3.6. Paris, France. Pp. 860-868.

Quinn, P.J., Carter, M.E., Marker, B. \& Carter, G.P., 1994. Mycobacterium species. In: Clinical Veterinary Microbiology, Wolfe Publishing, London. Pp. 156-170.

Shivaprasad, H.L and Palmieri C., 2012. Pathology of Mycobacteriosis in Birds. Vet. Clin. Exot. Anim., 15, 41-55

Sonaiya, E., Branckaert, R. \& Guèye, E., 1999. Research and development options for family poultry. In: Gueye E. F. (Ed) The First INFPD/FAO Electronic Conference 
on the Scope and Effect of Family Poultry Research and Development, (www.fao. org/ag/againfo/subjects/en/infpd/econf scope.html)

Dessie, T. \& Ogle, B., 2001. Poultry production systems in the central highlands of Ethiopia. Trop. Anim. Hlth. Prod., 33(6), 521-537.

Dessie, T., Kijora, C. \& Peters, K. J., 2003. Indigenous chicken ecotypes in Ethiopia: Growth and feed utilization potentials. Int. J. Poult. Sci., 2, 144-152.

Tadesse, S., Woldemeskel, M., Molla, B., Tibbo, M., Kidane, D., Medhin, G., and Britton, S., 2004. Avian mycobacteriosis in domestic chickens from selected agro-climatic regions in Ethiopia. Int. Appl. Res. Vet. Med., 2 (1), 17-25.

Tell, L., Leutenegger, C. and Larsen, R., 2003. Real-time polymerase chain reaction testing for the detection of Mycobacterium genavense and Mycobacterium avium complex species in avian samples. Avi. Dis., 47(4), 1406-1415.

Tell, L., Woods, L. and Cromie, R., 2001. Avian tuberculosis in birds. Rev. Sci. Tech. Off. Int. Epiz., 20, 180-203.

Thoen, C.O., 1997. Tuberculosis. In: Diseases of Poultry. $10^{\text {th }} e d$, Iowa State University Press, USA, Pp. 67-178.

Thrusfield, M., 2007. Veterinary epidemiology $3^{\text {rd }}$ ed., Blackwell Science Ltd, Oxford, UK, Pp. 178-197.

Zhu, L., Peng, Y., Ye, J., Wang, T., Bian, Z., Qin, Y., Zhang, H., and Ding, J., 2018. Isolation, identification, and characterization of a New Highly Pathogenic field isolate of Mycobacterium avium spp. avium. Front. Vet. Sci., 4, 243. 